\title{
IS07
}

\section{Interconnect Design in Support of Geophysical} Workloads

B. Bolding* (CRAY)

\section{SUMMARY}

Measurement of standard network performance metrics for high performance computing (HPC) has traditionally focused on quiet network bandwidth and latency. However, a key challenge in high performance networking is how to provide cost-effective, scalable global bandwidth, reflected, for instance, in the network's ability to sustain high levels of performance on complex geophysical workloads. We describe characteristics of network design that provide for a wide range of HPC-optimized system attributes. 